\title{
Tumor-to-Tumor Metastasis of Multiple Meningiomas and Clear Cell Renal Cell Carcinoma Metastasis as First Clinical Appearance of Kidney Cancer: A Case Report and Analysis
}

\author{
Johannes Dietterle ${ }^{10}$ Clara Frydrychowicz ${ }^{2} \quad$ Wolf Müller $^{2} \quad$ Karl-Titus Hoffmann $^{3}$ Katja Jähne ${ }^{1}$ \\ Jürgen Meixensberger ${ }^{1}$
}

${ }^{1}$ Department of Neurosurgery, University Hospital Leipzig, Leipzig, Germany

${ }^{2}$ Section of Neuropathology, Department of Diagnostic Medicine, University Hospital Leipzig, Leipzig, Germany

${ }^{3}$ Section of Neuroradiology, Department of Diagnostic Medicine, University Hospital Leipzig, Leipzig, Germany

\begin{abstract}
Address for correspondence Johannes Dietterle, MD, Department of Neurosurgery, University Hospital Leipzig, Liebigstraße 20, 04103 Leipzig, Germany (e-mail: johannes.dietterle@medizin.uni-leipzig.de).
\end{abstract}

J Neurol Surg Rep 2020;81:e10-e14.

\begin{abstract}
Keywords

- meningioma

- multiple meningiomas

- renal cell carcinoma

- tumor-to-tumor metastasis

Background Meningioma accounts for more than $35 \%$ of all diagnosed brain tumors of the central nervous system and, moreover, it is the most common benign recipient of tumor-to-tumor metastasis. Several cases with tumor-to-meningioma metastasis by breast, lung, and intestinal cancer have been described before.

Case description The case of a patient with a longstanding history of multiple meningiomas $(n=4)$ that suddenly became symptomatic and progressive in size is presented. Following extirpation of the two largest meningiomas, a histological examination revealed two separate tumor-to-meningioma metastases of clear cell renal cell carcinoma that was undiagnosed before. Post-surgical computed tomography scan then confirmed tumor-suspect lesions in both kidneys. After recovery and rehabilitation, adjuvant radio-chemo-therapy was applied according to protocols for kidney cancer. No other tumor-to-tumor-suspect event occurred since then for the remaining two meningiomas.

Conclusion Review of literature and our case strengthens the idea of meningioma as a favorable premetastatic niche. Considering that the patient lived with a stable disease for many years, a sudden progress of tumor size in association with neurological deterioration was highly suspected for malign involvement, including the possibility of tumor-to-tumor metastasis. Physicians should be aware about this phenomenon and treat patients accordingly to the underlying disease.
\end{abstract}

\section{Introduction}

Meningioma is a tumor originating from meningothelial cells and is mostly graded as World Health Organization grade I. ${ }^{1}$

received

April 23, 2019

accepted

September 28, 2019
DOI https://doi.org/

10.1055/s-0040-1708846. ISSN 2193-6358.
Accounting for $36.3 \%$ of all primary lesions of the central nervous system (CNS) and $53.0 \%$ of all benign primary brain tumors, it is the most common CNS tumor. ${ }^{2}$ Multiple meningiomas appear in around $10 \%$ of meningioma patients and
License terms

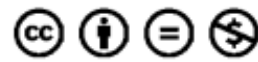

(c) 2020 Georg Thieme Verlag KG Stuttgart · New York 
are either sporadic or radiation-induced. Comparable to cases with single meningioma, management of multiple meningiomas mainly consists of surgery and surveillance. Surgery is recommended when a lesion becomes symptomatic or is progressive in size. ${ }^{3}$

Clear cell renal cell carcinoma (ccRCC) is the most common and most aggressive subtype of kidney cancer, accounting for around $\sim 80 \%$ of all cases. The median age at diagnosis is 64 years. If restricted to the kidney, surgery is performed with an average 5-year-survival rate of $92 \%$. However, if the disease spreads locally or systematically, only 12 to $67 \%$ of patients survive longer than 5 years. Therapy options for metastatic ccRCC stages are radiochemotherapy and, if applicable, surgery. ${ }^{4}$ In hope to improve the devastating survival rates, specific metabolic changes in glucose and lipid pathways of ccRCC might be promising targets for future systemic therapy. ${ }^{5}$

Also, tumor-to-meningioma metastasis of ccRCC has been described earlier. ${ }^{6}$ The first case of multiple central lesions as tumor-to-meningioma metastasis leading to the diagnosis of a presurgically unknown ccRCC is presented.

\section{Case Presentation}

\section{Medical History}

In May 2018, a 67 years old patient admitted to the department of neurosurgery with a sudden decrease in vigilance and worsened left hemiparesis. A cranial computed tomography (CT) scan showed multiple intracranial lesions. Her husband reported that she was first diagnosed with multiple meningiomas in 2008 when she suffered from an ischemic stroke that caused a mild residual hemiparesis on the left. Until 2011 she attended regular control appointments to monitor the intracranial tumors. Later, follow-up examinations were evaded.

\section{Clinical Examination}

The patient was in a reduced clinical condition (Karnofsky Performance Score 60-70). Sudden weight loss, night sweats, and fever were denied. She presented with GCS 14 (somnolent, but fully oriented) and left-sided hemiparesis. Meningism, nausea, or headache was not apparent. A seizure did not take place and the cranial nerves were deficit-free. Pupils appeared round and symmetrical and reacted to light bilaterally.

The physical examination of the cardiovascular system, lung, and abdomen brought no pathological findings. She took following daily medications: $1 \times 100 \mathrm{mg}$ acetylsalicylic acid, $1 \times 40 \mathrm{mg}$ pantoprazole, $1 \times 10 \mathrm{mg}$ amlodipine, $1 \times 160 \mathrm{mg}$ valsartan, $1 \times 6.25 \mathrm{mg}$ hydrochlorothiazide, and $2 \times 47.5 \mathrm{mg}$ metoprolol.

\section{Radiologic and Laboratory Diagnosis}

The cranial magnetic resonance imaging ( - Fig. $\mathbf{1}$ ) revealed the already known four meningiomas, three within the right brain hemisphere and one on the left side. Two lesions, parietal right and frontal left, presented perifocal edema and a significant progression in size when compared with scans from 2011.
Serum ionogram, hepatic, and renal function analyses were normal. Hematocrit was 0.401 , hemoglobin $14.2 \mathrm{~g} / \mathrm{dL}$, red blood cell count $5.1^{*} 10^{\wedge} 12 / \mathrm{L}$, and platelet count $184 \mathrm{G} / \mathrm{L}$. Leukocytes count was increased up to $16.01^{*} 10^{\wedge} 3 / \mu \mathrm{L}$ and Creactive protein slightly upregulated to $9.48 \mathrm{mg} / \mathrm{L}$ but no infectious focus could be identified.

\section{Therapy}

Already prior to the surgical procedure we began an oral therapy with dexamethasone to control perifocal brain edema. The right parietal meningioma with size progression was identified as a possible cause for the aggravated hemiparesis of the left body side. Therefore, a right frontoparietal osteoplastic craniotomy and microscopic tumor removal under ultrasound guidance was performed.

\section{Histology}

Histological examination revealed two distinct tumor morphologies. The first tumor featured meningothelial cells, with some psammoma bodies and whorl-shaped tumor cell growth, without increased mitotic activity. Immunohistochemistry of this tumor was positive for epithelial membrane antigen, somatostatin and vimentin, compatible with meningioma. The second neoplastic process was identified within and adjacent to the meningioma. Histomorphology and immunohistochemical profile strongly suggested a metastasis of a RCC. As typical morphological features, the tumor revealed lobulated growth and tumor cells with clear cytoplasm and distinct borders. The tumor expressed PAX8, CD10, and co-expressed cytokeratins (AE1/3) and vimentin; antigens typically found in renal cell carcinomas. Morphology and immunohistochemistry are summarized in -Fig. 2.

\section{Clinical Course}

A CT staging was performed after the histological diagnosis was received and revealed highly cancer suspect lesions in both kidneys. During the following 16 days the patient recovered from the procedure and was sent to rehabilitation.

Three weeks after release, she was again admitted to our department due to a recurrent decrease in vigilance. Our interdisciplinary tumor board recommended the removal of the second size-progressive lesion, located on the frontal left side. This lesion, too, was diagnosed as tumor-to-tumor metastasis (TTM) of the ccRCC. Again, the patient recovered and could be released after 22 days. During a control appointment at our outpatient center 6 weeks later, she presented with a generally improved clinical condition and radiation therapy was initiated.

\section{Discussion}

TTM is a well-known but rare phenomenon in oncology. Campbell et al were the first to define commonly accepted criteria to differentiate TTM from "collision tumors."7 Although collision tumors occur as two adjacently growing neoplasms of different origin that consecutively happen to infiltrate each other, TTM is defined by (1) exclusion of 


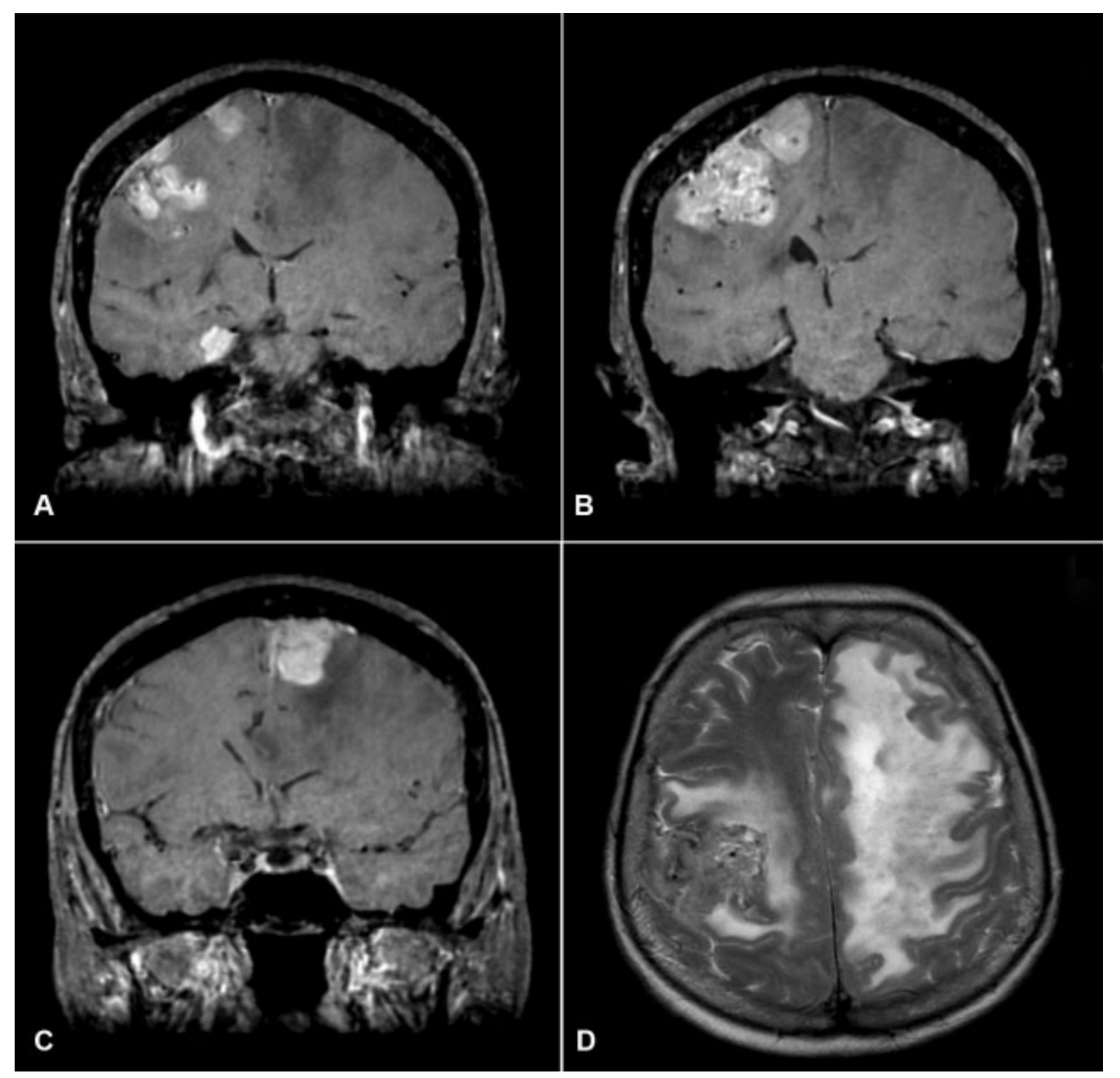

Fig. 1 (A-C): Post-contrast T1-weighted coronal brain magnetic resonance imagings demonstrating the four meningiomas. Three lesions originate from the convexity: two on the right (B), one the left side (C). The fourth tumor is attached to the base of right middle cranial fossa (A). (D) Axial T2-weighted image showing bilateral brain edema caused by the two size progressive lesions that were diagnosed as tumor-to-meningioma metastases of clear cell renal cell carcinoma.

lymphatic metastasis into lymphoma, (2) the histological proof of two distinct neoplasms, and (3) the enclosure of one tumor by at least a small rim of the other. RCC is considered to be the most common malignant recipient for this type of metastasis formation ${ }^{8}$ meningioma is the most common benign entity to be infiltrated by other neoplasms. ${ }^{9}$ Breast and lung cancer are known to be the most common donor malignancies for tumor-to-meningioma metastases, ${ }^{9-13}$ but many others were also reported before. ${ }^{9}$ To our knowledge, we here describe the first case where extirpation and histological analysis of multiple size-progressive meningiomas revealed metastases of previously undiagnosed ccRCC.

Theories about the origin of TTM were created since the first case of a tumor-to-meningioma case was reported in $1930 .^{14}$ To allow cancer cells within the bloodstream or lymphatic fluid to infiltrate tissue and grow, the right environment has to be created. The first to describe this interaction between cancer cells and stroma was Paget in 1889, defining the "seed and soil" theory. ${ }^{15}$ Today, this special constellation is called the "pre-metastatic niche" and characterized by immunosuppression, inflammation, angiogenesis and vascular permeability, lymphangiogenesis, organotropism, and reprogramming (metabolic, stromal, and epigenetic). ${ }^{16}$ Concerning meningioma, it has been demonstrated that tumor size seems to be associated with better blood perfusion ${ }^{17}$ and that overexpression of vascular endothelial growth factor in meningioma is associated with higher microvascular density. ${ }^{18}$ There is also evidence that macrophages in meningioma might promote inflammation as well as the permeability of brain-blood barrier, hence contributing to possible brain metastasis formation. ${ }^{19}$ In conclusion, meningioma seems to represent a favorable premetastatic niche.

\section{Conclusion}

Careful histological analysis of two extirpated central lesions led to the final diagnosis of ccRCC, supported by a post-surgical abdominal CT scan. Hence, the patient could be treated accordingly to protocols for kidney cancer. Clinical results and careful analysis of case reports prove that 

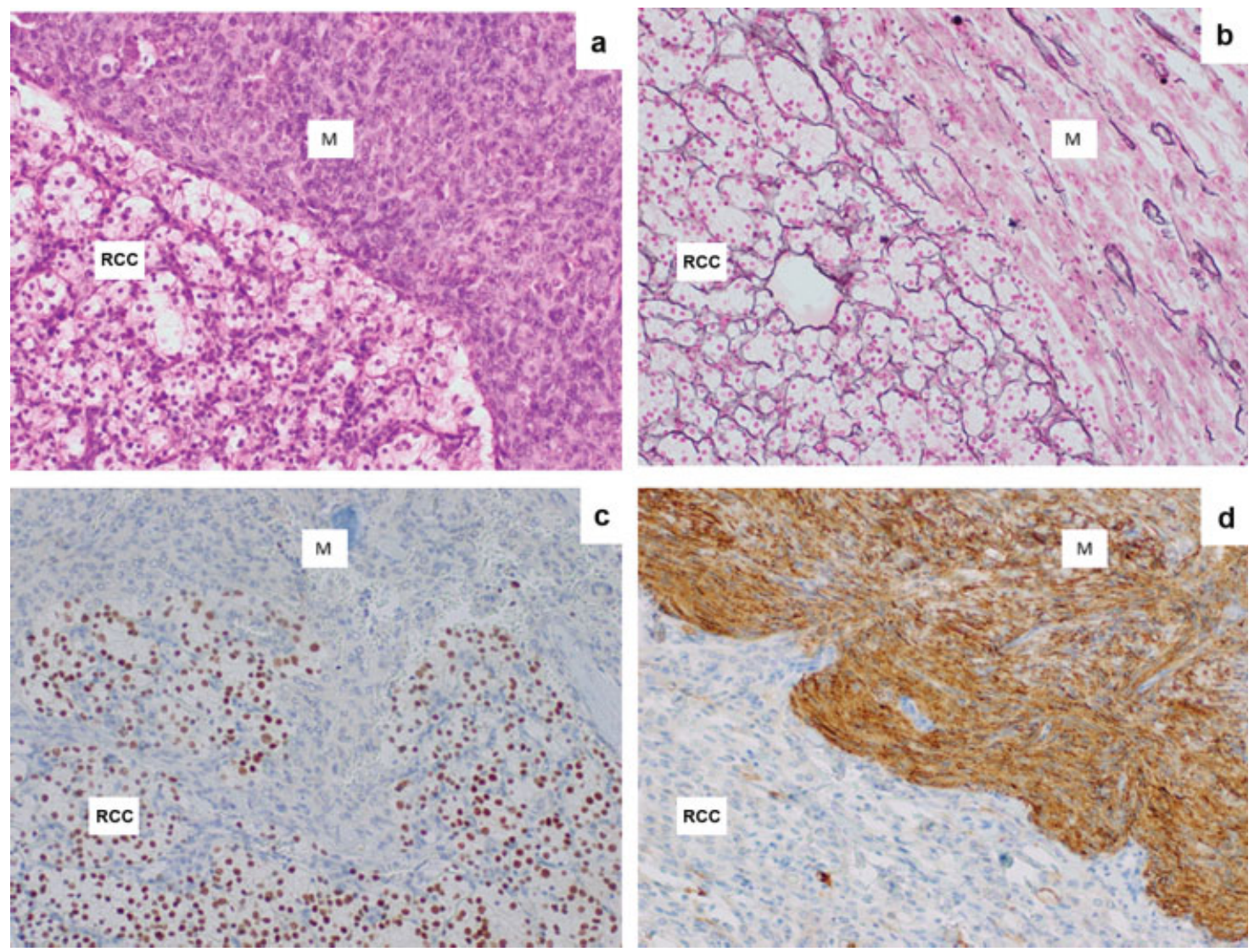

Fig. 2 (A) Hematoxylin and eosin (H\&E) staining of the collision tumor: histology shows typical features of metastatic renal cell carcinoma (RCC; left lower area), as well as typical features of meningioma (M; right upper area) (H\&E, x 200) (B) Gomori's silver impregnation staining technique for reticulin fibers of collision tumor, fine lobulated reticulin network in metastatic RCC (left lower area), compared with coarse in meningioma (M; right upper area) (TP, x 200). (C) Immunohistochemistry (IHC) detection of PAX8-positive cells of metastatic RCC (lower area) but not in meningioma (M; upper area) (PAX8, x200). (D) IHC results showed positive epithelial membrane antigen (EMA) staining in meningioma (M; right upper area); metastatic RCC was devoid of EMA (RCC; left lower area) (EMA, x 200).

meningioma seems to represent a favorable premetastatic niche. Our report with multiple tumor-to-meningioma metastases by ccRCC in one patient supports this hypothesis.

\section{Written Consent}

Written informed consent was obtained.

\section{Author's Contributions}

JD mainly wrote the manuscript with contributions by CF. KJ performed surgery, and JD assisted surgery. CF and WM performed pathologic classification of tumors. KTH provided radiological analysis. JM, KTH, and WM revised the manuscript. All authors read and approved the final manuscript.

\section{Conflict of Interest}

Authors declare that they have no conflict of interest.

\section{Acknowledgment}

We thank the patient for the permission to report her case. Furthermore, we acknowledge support from the German Research Foundation (DFG) and Leipzig University within the program of Open Access Publishing.

\section{References}

1 DeWitt JC, Mock A, Louis DN. The 2016 WHO classification of central nervous system tumors: what neurologists need to know. Curr Opin Neurol 2017;30(06):643-649

2 Ostrom QT, Gittleman H, Liao P, et al. CBTRUS Statistical Report: primary brain and other central nervous system tumors diagnosed in the United States in 2010-2014. Neuro-oncol 2017;19(5, suppl_5):v1-v88

3 Tsermoulas G, Turel MK, Wilcox JT, et al. Management of multiple meningiomas. J Neurosurg 2018;128(05):1403-1409

4 Motzer RJ, Jonasch E, Agarwal N, et al. Kidney Cancer, Version 2.2017, NCCN Clinical Practice Guidelines in Oncology. J Natl Compr Canc Netw 2017;15(06):804-834

5 Sanchez DJ, Simon MC. Genetic and metabolic hallmarks of clear cell renal cell carcinoma. Biochim Biophys Acta Rev Cancer 2018; 1870(01):23-31

6 Carr K, He L, Weaver K, Highfield Nickols H. Renal cell carcinoma metastatic to meningioma: tumor-to-tumor metastasis. Clin Neuropathol 2014;33(02):152-156

7 Campbell LV Jr, Gilbert E, Chamberlain CR Jr, Watne AL. Metastases of cancer to cancer. Cancer 1968;22(03):635-643

8 Sella A, Ro JY. Renal cell cancer: best recipient of tumor-to-tumor metastasis. Urology 1987;30(01):35-38

9 Schmitt HP. Metastases of malignant neoplasms to intracranial tumours: the "tumour-in-a-tumour" phenomenon. Virchows Arch A Pathol Anat Histopathol 1984;405(01):155-160

10 Takei H, Powell SZ. Tumor-to-tumor metastasis to the central nervous system. Neuropathology 2009;29(03):303-308 
e14 Tumor-to-Meningioma Metastases of Kidney Cancer Dietterle et al.

11 Ravnik J, Ravnik M, Bunc G, Glumbic I, Tobi-Veres E, Velnar T. Metastasis of an occult pulmonary carcinoma into meningioma: a case report. World J Surg Oncol 2015;13:292. Doi: 10.1186/ s12957-015-0714-3

12 Pham JT, Kim RC, Nguyen A, et al. Intracranial meningioma with carcinoma tumor-to-tumor metastasis: two case reports. CNS Oncol 2018;7(02):CNS09. Doi: 10.2217/cns-2017-0022

13 Farrag A, Ansari J, Ali M, Sunbuli G, Kassem H, Al Hamad A-A. Intracranial meningioma as primary presentation for an undiagnosed collision metastatic breast cancer: case report and literature review. Mol Clin Oncol 2018;8(05):661-664

14 Fried BM. Metastatic inoculation of a meningioma by cancer cells from a bronchiogenic carcinoma. Am J Pathol 1930;6(01): $47-52.1$
15 Paget S. The distribution of secondary growths in cancer of the breast. 1889. Cancer Metastasis Rev 1989;8(02):98-101

16 Peinado H, Zhang H, Matei IR, et al. Pre-metastatic niches: organspecific homes for metastases. Nat Rev Cancer 2017;17(05):302-317

17 Karsy M, Burnett B, Di leva A, Cusimano MD, Jensen RL. Microvascularization of Grade I meningiomas: effect on tumor volume, blood loss, and patient outcome. J Neurosurg 2018;128(03):657-666

18 Dharmalingam P, Roopesh Kumar VR, Verma SK. Vascular endothelial growth factor expression and angiogenesis in various grades and subtypes of meningioma. Indian J Pathol Microbiol 2013;56(04):349-354

19 Polyzoidis S, Koletsa T, Panagiotidou S, Ashkan K, Theoharides TC. Mast cells in meningiomas and brain inflammation. J Neuroinflammation 2015;12:170 